\title{
APLICACIÓN DE TÉCNICAS DE ANÁLISIS MULTICRITERIO A LA LOCALIZACIÓN ÓPTIMA DE EXTRACCIONES DE ARENAS Y GRAVAS EN EL ENTORNO DE ZARAGOZA
}

\author{
M.T. Lamelas Gracia \\ Centro Universitario de la Defensa de Zaragoza. España \\ GEOFOREST - IUCA, Departamento de Geografía y Ordenación del Territorio. Universidad de Zaragoza \\ tlamelas@unizar.es
}

\section{RESUMEN}

La toma de decisiones sobre los usos del suelo es, en si misma, un problema multicriterio. En este artículo se comparan diferentes metodologías de análisis multicriterio para identificar la mejor localización espacial de extracciones de arenas y gravas en el entorno de Zaragoza (España). Se ha llevado a cabo un análisis o búsqueda de localización óptima mediante la metodología SAW y se ha utilizado la metodología PROMETHEE-2 para llevar a cabo un análisis de alternativa óptima. Finalmente, para tener cierto control en la incertidumbre de los valores de entrada, se ha realizado una aproximación estocástica. El principal resultado es la similitud en los modelos obtenidos al aplicar diferentes metodologías.

Palabras clave: Ordenación del territorio, SDSS, SAW, AHP, PROMETHEE, Desarrollo sostenible.

\section{ABSTRACT}

Land-use decision making is an intrinsic multicriteria problem. In this article we compare different multicriteria methodologies to identify the most appropriate spatial pattern for future sand and gravel extraction in Zaragoza (Spain). A site search analysis is performed using a

Fecha de recepción: septiembre 2012.

Fecha de aceptación: junio 2014. 
SAW method and PROMETHEE-2 is used to perform a site selection analysis. Finally, in order to account for uncertainty in the input values we also apply a stochastic approach. The main result is the similarity of the results obtained after applying the different approaches.

Key words: Land-use management, SDSS, SAW, AHP, PROMETHEE, sustainable development.

\section{INTRODUCCIÓN}

La legislación europea apela a un desarrollo bien planificado y sostenible. El objetivo general debería ser mejorar o cambiar el proceso de toma de decisiones, para que la consideración de asuntos socioeconómicos y medio ambientales se integre completamente en el mismo y se asegure la participación pública. Asimismo, la Estrategia Española de Desarrollo Sostenible (EEDS) plantea como eje transversal el impulsar la participación ciudadana, de las empresas y de los interlocutores sociales en los procesos de toma de decisiones.

Por tanto, cualquier proceso de planeamiento debe orientarse a la incorporación de una mezcla de información objetiva (estimaciones cuantitativas, encuestas, etc.) y subjetiva (preferencias, prioridades, juicios, etc. de los centros de decisión). La idea de combinar los elementos subjetivos y objetivos de todo proceso de planeamiento en un sistema informatizado recae sobre el concepto de Sistema de Ayuda a la Decisión Espacial (Spatial Decision Support System SDSS) (Booty et al., 2001; Malczewski, 2004; Shim et al., 2002).

Estos sistemas pueden ser definidos como la combinación de metodologías sofisticadas para el apoyo a la toma de decisiones (técnicas de evaluación multicriterio - EMC) y Sistemas de Información Geográfica - SIG (Gómez-Delgado y Barredo, 2005; Jankowski, 1995; Malczewski, 2004; Marinoni, 2005), y por esta razón son herramientas óptimas para el planeamiento y desarrollo sostenible de zonas urbanas.

Aunque la consagración del análisis multicriterio se realiza a partir de los años 70, es a partir aproximadamente del año 1991 (Gómez-Delgado y Barredo, 2005), en la denominada cuarta etapa de evolución en los SIG, cuando se desarrollan los análisis de decisión. Los trabajos de Voogd (1983), Janssen y Rietveld (1990), Carver (1991), Can (1993), Pereira y Duckstein (1993), Barredo y Bosque (1995), Bosque y Franco (2000), Bosque y Moreno (2004), Bosque et al. (1999), Thill (1999), Eastman et al., (1993), entre mucho otros, indican la tendencia de integración de técnicas de Evaluación Muliticriterio (EMC) y SIG, tratando de solventar de algún modo las carencias analíticas de los SIG (Gómez-Delgado y Barredo, 2005). Wallenius et al. (2008), hacen un estudio de la evolución de la utilización de las técnicas de EMC desde el año 1992 hasta el 2006, revelando que la utilización de técnicas multiatributo se ha incrementado en 4,2 veces en este periodo.

En los últimos años se ha realizado un gran esfuerzo en la integración de técnicas de EMC y GIS en Internet (ver Carver 1999; Rinner, 2003; Rinner y Malczewski, 2002; Zhu y Dale, 2001).

Desde nuestro punto de vista, la toma de decisiones sobre los usos del suelo es, en si mismo, un problema de decisión multicriterio. Por esta razón consideramos que los métodos de EMC son válidos para apoyar al proceso de toma de decisiones por medio del análisis de la idoneidad del terreno para acoger diferentes usos del suelo. 
Además, para cumplir las funciones de Ordenación del Territorio, los instrumentos a utilizar deben ser actualizables, multi-escalares y deben contener una amplia gama de informaciones relativas al medio ambiente; es decir, al medio físico, medio biótico, aspectos antrópicos y sus interrelaciones. Desde este punto de vista, los Sistemas de Información Geográfica (SIG) son enormemente necesarios (Amadio et al., 2002, Bosque y García, 2000).

Existen en la bibliografía científica diferentes intentos de clasificar los métodos de decisión multicriterio por diversos autores (Gómez-Delgado y Barredo, 2005, Jankowski, 1995; Malczewski, 1999; Pereira y Duckstein, 1993; Vincke, 1986; Voogd, 1983). La mayoría coincide en que las reglas aditivas de decisión (Sumatoria lineal ponderada, Análisis de la concordancia, Jerarquías analíticas, etc.) son los métodos para la toma de decisión multiatributo más conocidos y ampliamente utilizados en el proceso de toma de decisiones basado en la utilización de SIG.

En este artículo se comparan los resultados obtenidos tras llevar a cabo dos análisis distintos de la capacidad o idoneidad del terreno para acoger extracciones de gravas y arenas, aplicando diferentes técnicas de análisis multicriterio. El análisis multicriterio se ha llevado acabo en un entorno ráster con dos objetivos bien diferenciados. En el primero de ellos, análisis de localización óptima, cada pixel en la zona de estudio representaba una posible alternativa de localización. Este análisis se ha desarrollado utilizando la metodología SAW que implica una suma ponderada, de manera que puede ser fácilmente implementado en SIG (Lamelas, 2007; Lamelas et al., 2010). En el segundo objetivo, análisis de alternativa óptima, se ha utilizado la metodología PROMETHEE-2 (Brans et al., 1986) y una serie de alternativas de localización predefinidas (Lamelas, 2007, Lamelas et al., 2010). Las diferentes técnicas utilizadas en el proyecto han sido desarrolladas e integradas en ArcGIS por Marinoni (2004, 2005).

Uno de los problemas y críticas en la utilización de técnicas multicriterio es la necesidad de asignar pesos a los criterios. Existe una gran variedad de aproximaciones para llevar a cabo dicha asignación (Malczewski, 1999), aunque probablemente la más conocida es AHP, que también ha sido utilizada en este trabajo.

Otro requerimiento de las técnicas de EMC, es la asignación de un valor a los criterios, que es, en algunas ocasiones, subjetiva. La información que ha sido capturada de manera directa suele ser acertadamente considerada como más fiable que la información que ha sido estimada, interpolada, extraída de un mapa o simplemente interpretada. Por esta razón la manera de obtener la información relativa a los criterios juega un papel central (Marinoni, 2005). Una forma de resolver este dilema puede ser la utilización de una aproximación estocástica que tiene en cuenta la incertidumbre en los valores asignados a los criterios y que es presentada al final de este artículo.

\section{ANTECEDENTES Y METODOLOGÍA}

\section{1. Área de estudio y antecedentes del proyecto}

La ciudad de Zaragoza está situada en el corredor del Ebro, un sector económico, extremadamente dinámico, localizado en el noreste de la Península Ibérica (Figura 1). El clima 
de este sector presenta características semiáridas, una precipitación media anual de unos 350 $\mathrm{mm}$ y una temperatura media anual de aproximadamente $15^{\circ} \mathrm{C}$.

Desde el punto de vista geológico, el relleno terciario, sedimentario, continental de la Cuenca del Ebro está compuesto por conglomerados y areniscas en los márgenes, pasando por arcillas, margas, evaporitas y carbonatos hacia el centro de la Cuenca (Benito et al., 1998). En el sector central de la Depresión, los depósitos de playa-lake conforman uno de los mayores afloramientos de yesos en el área, sólo cubierto en algunos sectores por los diferentes glacis y terrazas depositados durante el Cuaternario por el río Ebro y sus afluentes (Gállego, Huerva, Jalón y Ginel), conformando un acuífero aluvial que hace de la karstificación un proceso activo y permanente. Además, este acuífero cuaternario es un recurso importante tanto por su carácter de reserva de agua subterránea para el uso doméstico, industrial y agrícola, como de reserva de arenas y gravas para el abastecimiento de materiales a la ingeniería civil.

Precisamente, ha sido la disponibilidad de estos recursos la causante del rápido desarrollo de la ciudad cuyas infraestructuras han provocado algunos impactos en el medioambiente. En este sentido, también la intensificación del regadío ha provocado la subsidencia del terreno, que, en definitiva, ha producido daños y/o destrucción en carreteras, edificios y redes de abastecimiento de gas y agua (Soriano y Simón, 1995). Del mismo modo, muchas infraestructuras han ocupado suelos de gran fertilidad, imposibilitando su uso agrícola, o sobre zonas de alto valor ecológico, afectándolas de forma negativa e irrecuperable (Lamelas, 2007).

De todo lo anterior se deduce que el entorno de la ciudad de Zaragoza requiere de un exhaustivo análisis de factores geocientíficos, como es la regionalización de sus georrecursos y, en la medida de lo posible, sus georriesgos. Entendiendo como aspectos geocientíficos los relativos a las Ciencias de la Tierra (Geología, Geomorfología, Hidrología, entre otras) que estudian la estructura interna, la morfología y la dinámica superficial y la evolución del planeta Tierra. Por este motivo, se inició un proyecto cuyo principal objetivo es el desarrollo de un esquema metodológico que facilitara la toma de decisiones sobre diferentes formas de uso del suelo, teniendo en cuenta estos aspectos geocientíficos (georrecursos y georriesgos), y posibilitando el desarrollo sostenible de un entorno urbano en crecimiento. El propósito era llevar a cabo un análisis de la idoneidad o capacidad del terreno para acoger determinados usos del suelo, con objeto de identificar los más apropiados para el futuro, de acuerdo con las preferencias específicas del desarrollo sostenible. Este objetivo implica la realización de los siguientes pasos:

- Caracterización de la zona de estudio utilizando SIG.

- Modelización de georrecursos y georriesgos con la ayuda de SIG y modelización 2D y $3 \mathrm{D}$.

- Análisis de la capacidad del terreno para acoger un determinado uso del suelo mediante SDSS.

Este artículo se centra en el análisis de la capacidad del terreno para acoger zonas de extracción de arenas y gravas. Como se ha mencionado con anterioridad, se comparan los resultados obtenidos tras llevar a cabo dos análisis utilizando diferentes técnicas de análisis multicriterio. Para más información acerca del esquema general del proyecto y de la modelización de georrecursos y georriesgos se pude consultar Lamelas et al. (2010) y Lamelas (2007). 
Figura 1

LOCALIZACIÓN Y GEOMORFOLOGÍA DEL ÁREA DE ESTUDIO

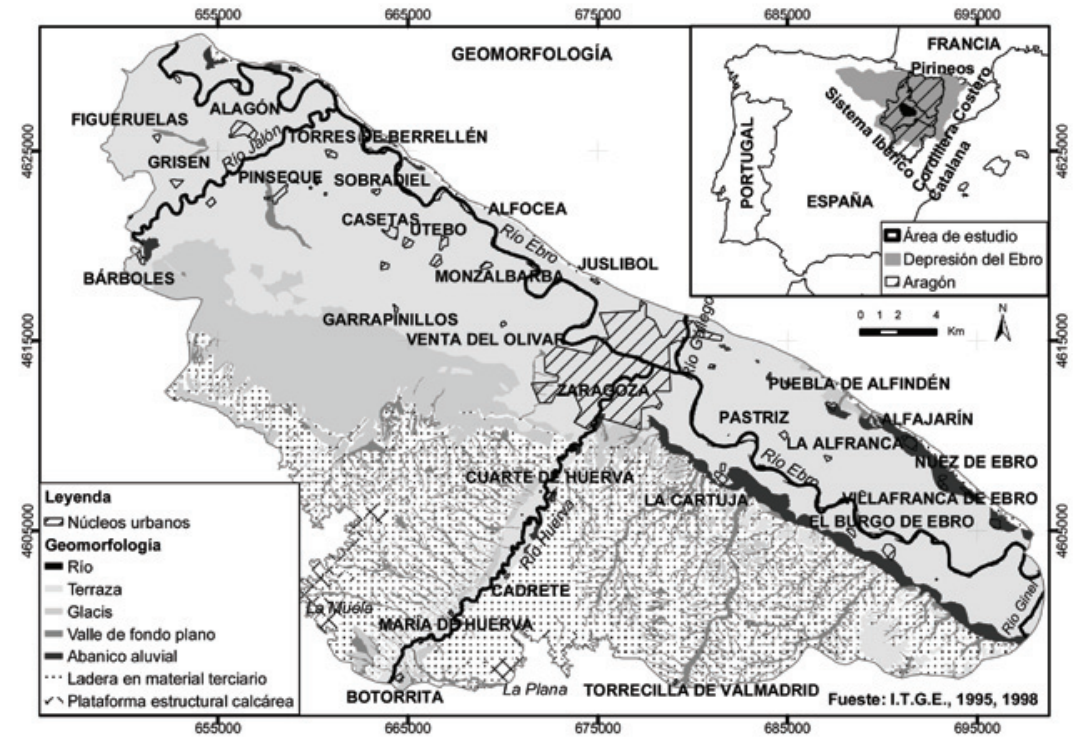

\section{Metodología}

En el contexto del análisis de la idoneidad o capacidad del terreno para determinados usos del suelo es importante diferenciar entre el problema de la selección de alternativa óptima (site selection problem) y el problema de búsqueda de localización óptima (site search problem). El propósito del análisis de alternativa óptima es identificar la mejor localización para una determinada actividad, dado un grupo de localizaciones posibles. El problema es ordenar las alternativas de localización por preferencia, basándose en sus características, de manera que se identifica la mejor localización. En el caso de que no exista un grupo determinado de alternativas, el problema se define como un análisis de localización óptima (Malczewski, 2004).

Con respecto a los métodos aplicados en este trabajo, la principal ventaja de la metodología SAW, es su bajo grado de complejidad que la hace muy atractiva para el análisis de localización óptima. Es precisamente esta simplicidad, la que hace que la suma ponderada sea actualmente una de las técnicas más ampliamente utilizadas en casos reales (Eastman, 1997; Heywood et al., 2002; Janssen y Rietveld, 1990; Malczewski y Rinner, 2005).

En el caso del análisis de alternativa óptima se ha utilizado la metodología PROMETHEE-2 que pertenece a la familia de métodos de concordancia (outranking techniques). Estas técnicas requieren la comparación por pares de todas las alternativas, lo que es obviamente impracticable para aplicaciones donde el número de alternativas/píxeles en una base de datos es del orden de decenas y cientos de miles (Pereira y Duckstein, 1993). Para obtener información más detallada acerca de las dos metodologías utilizadas, se puede consultar Saaty (1977), Brans et al. (1986), Lamelas et al. (2010) y Lamelas (2007). 
Para llevar a cabo las dos aproximaciones; el análisis de localización óptima, donde cada píxel representa una alternativa de localización, y análisis de alternativa óptima, se deben seguir varios pasos:

- Definición de alternativas: posibles localizaciones.

- Definición de limitantes: áreas con restricciones para un determinado uso.

- Definición de los criterios: factores que tienen un papel importante en el proceso de decisión.

- Asignación de pesos de decisión.

Los pesos de los criterios han sido determinados mediante la metodología AHP. Esta técnica requiere la comparación por pares de criterios, donde es preciso asignar valores numéricos que expresen la preferencia de un criterio frente a otro. Con posterioridad, los valores asignados son sintetizados para determinar el orden de preferencia de los criterios en términos de valores numéricos que equivalen a los pesos de los criterios. Aplicaciones empíricas sugieren que este método de comparación por pares es una de las técnicas más efectivas para la toma de decisiones espaciales basadas en la utilización de SIG (Eastman et al., 1993; Malczewski et al., 1997). Existen una gran cantidad de ejemplos bien documentados de la aplicación de este método con éxito (Banai, 1993; Banai-Kashani, 1989; Malczewski, 1996; Miller et al., 1998).

En numerosas ocasiones, la información utilizada para llevar a cabo un procedimiento de análisis multicriterio presenta inexactitud, imprecisión y ambigüedad. No obstante, la gran mayoría de metodologías asume que la información de entrada es precisa y exacta. Existen en la literatura científica algunos intentos de solución que combinan procedimientos de EMC en SIG con análisis de sensibilidad (Lodwick et al., 1990) y análisis de propagación del error (Hevelink et al., 1989). Otra alternativa es usar métodos basados en la lógica difusa (Malczewski, 2004).

Además, la elección de los valores de entrada en un procedimiento de análisis multicriterio no es siempre sencilla, ya que los valores de los criterios para cada una de las diferentes alternativas no siempre son únicos sino que pueden presentar un rango de valores (Marinoni, 2005). El realizar el análisis utilizando el valor medio de los datos produce como resultado un valor medio, pero la incertidumbre tanto en los valores de entrada como en los resultados obtenidos no puede ser cuantificada. Una posible solución consiste en llevar a cabo una aproximación estocástica que utilice distribuciones de probabilidad para generar los valores de entrada en el análisis, en lugar de utilizar un valor único. Esta aproximación requiere la repetición del análisis, múltiples veces, con diferentes valores de entrada para los criterios. Los valores de entrada se obtienen a partir de las distribuciones de probabilidad que se asignan a los datos tomados empíricamente. Esta aproximación utiliza todo el rango de posibles valores de los criterios y los casos extremos son representados como eventos raros de forma más realística, de acuerdo con su baja probabilidad de aparición.

\section{Análisis de localización óptima de extracciones}

En este análisis cada pixel de la zona de estudio es considerado como una alternativa de localización. Los limitantes para la localización, se refieren a zonas en las que la actividad extractiva no está permitida. Estas restricciones de uso se deben en algunos casos a la existencia de otros usos 
(e.g. uso residencial) y en otros casos a la existencia de protecciones medioambientales o restricciones de uso debidas a la planificación del territorio. Estas restricciones de uso son:

- Espacio Natural Protegido: Reserva Natural de los Galachos de La Alfranca, Pastriz, La Cartuja y El Burgo de Ebro.

- Espacios urbanizados: Cartografiados a partir del Mapa Topográfico Nacional (MTN), a escala 1:25.000, del Instituto Geográfico Nacional (IGN).

- Infraestructuras y sus áreas de protección: Carreteras, vías de tren, canales, etc. Cartografiadas a partir del MTN a escala 1:25.000.

- Planificación: Plan General de Ordenación Urbana de Zaragoza (PGOUZ) y Plan de Ordenación de los Recursos Naturales (PORN) del Ebro.

- Vías Pecuarias: Reguladas por la LEY 10/2005, de 11 de noviembre, de Vías Pecuarias de Aragón y cartografiadas a partir de información proveniente del Gobierno de Aragón (DGA).

- Polígonos industriales: Información proveniente del Instituto Aragonés de Fomento (IAF) de la DGA.

- Zonas ya extraídas: Información proporcionada por el Ayuntamiento de Zaragoza y el Servicio de minas del Servicio Provincial de Industria del Gobierno de Aragón.

Se tuvieron en consideración una gran variedad de factores sociales, económicos y medioambientales, ya que nuestro principal objetivo es el promover un desarrollo sostenible, evitando, en la medida de los posible, la destrucción de georrecursos y la ocurrencia de catástrofes asociadas a georriesgos. La Figura 2 muestra la cartografía de todas las variables. Estas variables son:

- Afección a espacios naturales de importancia: debe evitarse la afección a Zonas de Especial Protección para las Aves (ZEPAS), Hábitats, Puntos de Interés Geológico, y otras áreas naturales, consideradas como un recurso muy valioso, cuya cartografía ha sido cedida, principalmente, por el Gobierno de Aragón. Además, la ley de evaluación de impacto ambiental indica que las extracciones deben localizarse a una distancia mayor de $2 \mathrm{~km}$ de estas zonas.

- Protección del agua subterránea: análisis del terreno y modelización realizado en este mismo proyecto mediante la utilización del modelo de Hölting et al. (1995), desarrollado por el Servicio Geológico Alemán, integrado en SIG (Lamelas, 2007). Este factor es considerado en el análisis ya que la extracción de arenas y gravas implica una reducción en la protección del acuífero, por lo que debe evitar explotarse las zonas con una alta vulnerabilidad a la contaminación del acuífero, para evitar los riesgos asociados a la misma.

- Capacidad agrícola de los suelos: cartografía realizada mediante la aplicación del Modelo Cervatana (de la Rosa y Magaldi, 1982) en SIG para este mismo proyecto (Lamelas, 2007). Se trata de un modelo cualitativo para evaluar la capacidad general agrícola de los suelos que utiliza los siguientes factores: relieve, suelos, clima y cubierta del suelo. Este factor se ha incluido ya que se considera que los suelos de mayor fertilidad deben ser conservados para su uso agrícola.

- Distancia a carreteras: la ley de evaluación de impacto ambiental indica que las extracciones deben localizarse a una distancia mayor de $2 \mathrm{~km}$ de vías de comunicación para evitar el impacto paisajístico que generan este tipo de instalaciones. 
- Distancia a núcleos de población: según la citada Ley la distancia de las extracciones a núcleos de población de más de 1.000 habitantes debe ser superior a $2 \mathrm{~km}$.

- Distancia a zonas de extracción existentes: esta distancia no debe ser inferior a $5 \mathrm{~km}$ de acuerdo con la misma Ley para evitar la concentración espacial de la actividad.

- Nivel freático: está prohibido por normativa explotar una extracción en los momentos en los que el nivel freático alcanza la superficie, por lo que las zonas en las que el nivel freático es más profundo son más rentables ya que la capacidad del recurso se incrementa. El modelo del nivel freático utilizado es el desarrollado por Lamelas (2007) para la modelización de la protección del agua subterránea.

- Localización del georrecurso: se ha utilizado un modelo del espesor de los depósitos de arenas y gravas elaborado en el proyecto a partir de un modelo geológico tridimensional de los depósitos cuaternarios realizado en Gocad (Lamelas, 2007; Lamelas et al., 2010).

- Espesor del material sobrante: elaborado a partir del modelo 3D citado con anterioridad. Este factor es utilizado ya que la extracción de este material es costosa, de manera que son más apropiadas las zonas que presentan un menor espesor del mismo.

Figura 2

CARTOGRAFÍA DE VARIABLES. 1) DISTANCIA ZONAS NATURALES PROTEGIDAS, 2) DISTANCIAA EXTRACCIONES, 3) DISTANCIAA NÚCLEOS DE POBLACIÓN, 4) DISTANCIAA CARRETERAS, 5) NIVEL FREÁTICO, 6) PROTECCIÓN DEL ACUÍFERO, 7) CAPACIDAD AGRÍCOLA DE LOS SUELOS, 8) ESPESOR DEL RECURSO,

9) ESPESOR DEL MATERIAL SOBRANTE
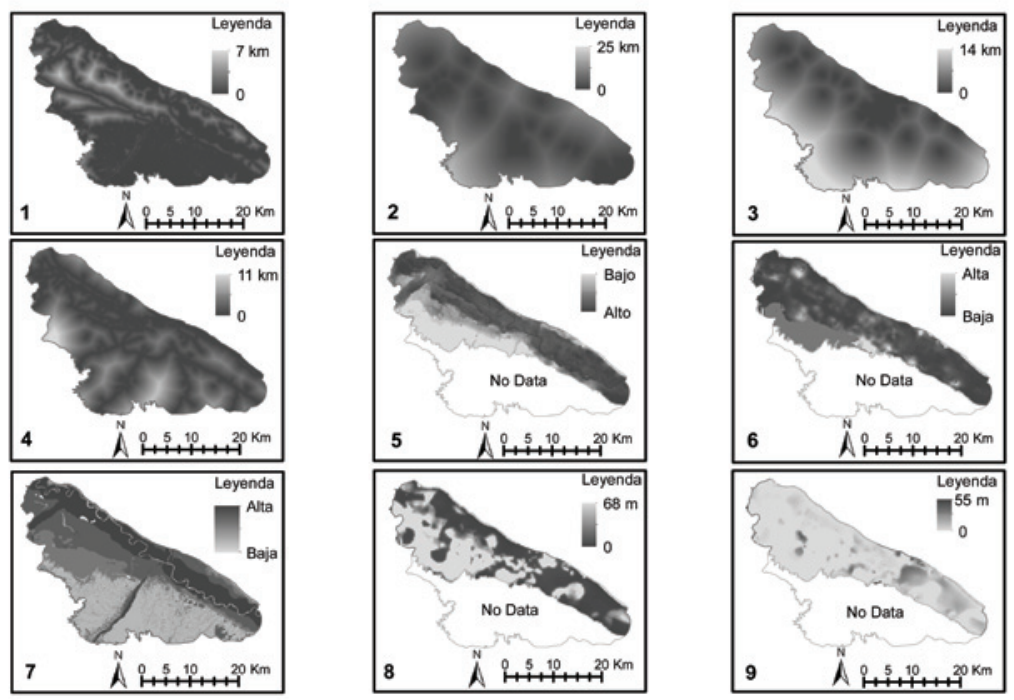

Muchas de las metodologías de EMC requieren que las variables sean estandarizadas para su incorporación como criterios en el proceso de localización óptima. Esta estandarización asegura el que los pesos de los criterios reflejen apropiadamente su importancia. En nuestro caso, el método de estandarización utilizado puede incluirse en la aproximación de escalas subjetivas (Malczewiski, 2004) ya que las variables se clasifican de acuerdo a unos rangos subjetivos. Estos 
rangos se determinan siguiendo, en algunos casos, las indicaciones de las Leyes medioambientales o, en otros casos, los rangos ya establecidos en los modelos desarrollados en el proyecto. De esta forma, las variables se han clasificado en 6 categorías: valores de 1 a 3 representan los peores valores de idoneidad, mientras que los valores de 4 a 6 representan los más óptimos.

La elección de 6 categorías para la estandarización de las variables es consecuencia de la adaptación de este número de clases a las variables introducidas ya que el mayor número de clases en los modelos que serán introducidos en el análisis es 5. Es necesario reservar una categoría para las zonas fuera de los modelos, en el caso de modelos que no cubren la totalidad de la zona de estudio. Éste es el caso de la susceptibilidad del terreno a la formación de dolinas y la protección del agua subterránea, entre otros. En estos dos casos, por ejemplo, el mayor valor de idoneidad (valor 6) ha sido asignado a las zonas fuera del modelo ya que la protección del agua subterránea es mayor en las zonas localizadas fuera del Acuífero Cuaternario y, en el caso del desarrollo de dolinas, la mayoría de los expertos convienen que las zonas más peligrosas son las zonas de Karst cubierto, en donde los depósitos cuaternarios se apoyan directamente sobre los depósitos terciarios.

En las Tablas 1-8 se puede consultar los valores asignados a los criterios en función de los valores originales de los modelos y cartografías. Para más información acerca del proceso de estandarización se puede consultar (Lamelas, 2007; Lamelas et al., 2010).

Los pesos de los criterios han sido asignados con la ayuda del AHP integrado en ArcGIS, herramienta desarrollada por el Instituto de Geociencias Aplicadas de la Universidad Tecnológica de Darmstadt (Marinoni, 2004). Esta herramienta puede ser descargada directamente de la página Web de ESRI (http://arcscripts.esri.com/). Para más información acerca del funcionamiento de la metodología AHP se puede consultar (Lamelas, 2007; Lamelas et al., 2010).

En el caso de la localización de extracciones de arenas y gravas, los valores de preferencia de los criterios se han asignado tras entablar varias conversaciones con expertos en el tema del Ayuntamiento de Zaragoza, el Gobierno de Aragón y la Confederación Hidrográfica del Ebro.

En un último paso, todos los factores clasificados (criterios), archivos ráster, son multiplicados por sus correspondientes pesos y son sumados.

Además, para comprobar la robustez de los resultados se ha llevado a cabo un análisis de la sensibilidad del modelo que ha consistido en la modificación del objetivo final hacía la obtención de un desarrollo que preste mayor importancia al beneficio económico y cambiando, por tanto, las preferencias en los criterios y los pesos asignados a los mismos.

Tabla 1

VALORES UTILIZADOS EN LA ESTANDARIZACIÓN DE LAS ZONAS NATURALES PROTEGIDAS

\begin{tabular}{|l|c|}
\hline \multicolumn{1}{|c|}{ Valor original } & Valor estandarizado \\
\hline Zonas incluidas dentro de los espacios protegidos & 1 \\
\hline Distancia de $0-500 \mathrm{~m}$ de zonas protegidas & 2 \\
\hline Distancia de $500-1.000 \mathrm{~m}$ de zonas protegidas & 3 \\
\hline Distancia de $1.000-2.000 \mathrm{~m}$ de zonas protegidas & 4 \\
\hline Distancia de $2.000-4.000 \mathrm{~m}$ de zonas protegidas & 5 \\
\hline Zonas a más de $4.000 \mathrm{~m}$ de distancia de zonas protegidas & 6 \\
\hline
\end{tabular}


Tabla 2

VALORES UTILIZADOS EN LA ESTANDARIZACIÓN DE LA PROTECCIÓN DEL AGUA SUBTERRÁNEA. VALORES EN FUNCIÓN DE LA CARTOGRAFÍA REALIZADA SIGUIENDO EL MODELO DE HÖLTING ET AL. (1995) INTEGRADO EN SIG (LAMELAS, 2007)

\begin{tabular}{|l|c|}
\hline \multicolumn{1}{|c|}{ Valor original } & $\begin{array}{c}\text { Valor } \\
\text { estandarizado }\end{array}$ \\
\hline Protección del acuífero: 0-500 & 1 \\
\hline Protección del acuífero: 500-1000 & 2 \\
\hline Protección del acuífero: $1000-2000$ & 3 \\
\hline Protección del acuífero: 2000-4000 & 4 \\
\hline Protección del acuífero: $>4000$ & 5 \\
\hline Zonas fuera del modelo & 6 \\
\hline
\end{tabular}

Tabla 3

VALORES UTILIZADOS EN LA ESTANDARIZACIÓN DE LA CAPACIDAD AGRÍCOLA DE LOS SUELOS. VALORES EN FUNCIÓN DE LA CARTOGRAFÍA REALIZADA SIGUIENDO EL MODELO CERVATANA (LAMELAS, 2007)

\begin{tabular}{|l|c|}
\hline \multicolumn{1}{|c|}{ Valor original } & \multicolumn{1}{|c|}{$\begin{array}{c}\text { Valor } \\
\text { estandarizado }\end{array}$} \\
\hline Areas sin suelo & 1 \\
\hline $\begin{array}{l}\text { Moderada capacidad agrícola con } \\
\text { la deficiencia climática como factor } \\
\text { limitante }\end{array}$ & 3 \\
\hline $\begin{array}{l}\text { Moderada capacidad agrícola con la } \\
\text { deficiencia climática y el suelo como } \\
\text { factores limitantes }\end{array}$ & 4 \\
\hline $\begin{array}{l}\text { Moderada capacidad agrícola con la } \\
\text { deficiencia climática, el suelo y la } \\
\text { pendiente como factores limitantes }\end{array}$ & 5 \\
\hline
\end{tabular}

Tabla 4

VALORES UTILIZADOS EN LA ESTANDARIZACIÓN DE LA DISTANCIAA CARRETERAS Y LA DISTANCIAA NÚCLEOS URBANOS. RANGOS ESTABLECIDOS TENIENDO EN CUENTA LA LEY DE EVALUACIÓN DE IMPACTO AMBIENTAL

\begin{tabular}{|l|c|}
\hline \multicolumn{1}{|c|}{ Valor original } & $\begin{array}{c}\text { Valor } \\
\text { estandarizado }\end{array}$ \\
\hline $0-500 \mathrm{~m}$ & 1 \\
\hline $500-1000 \mathrm{~m}$ & 2 \\
\hline $1000-2000 \mathrm{~m}$ & 3 \\
\hline $2000-3000 \mathrm{~m}$ & 4 \\
\hline $3000-4000 \mathrm{~m}$ & 5 \\
\hline$>4000 \mathrm{~m}$ & 6 \\
\hline
\end{tabular}


Tabla 5

VALORES UTILIZADOS EN LA ESTANDARIZACIÓN DE LA DISTANCIAA ZONAS DE EXTRACCIÓN EXISTENTES. RANGOS ESTABLECIDOS TENIENDO EN CUENTA LA LEY DE EVALUACIÓN DE IMPACTO AMBIENTAL

\begin{tabular}{|l|c|}
\hline \multicolumn{1}{|c|}{ Valor original } & Valor estandarizado \\
\hline $0-1000 \mathrm{~m}$ & 1 \\
\hline $1000-3000 \mathrm{~m}$ & 2 \\
\hline $3000-5000 \mathrm{~m}$ & 3 \\
\hline $5000-7000 \mathrm{~m}$ & 4 \\
\hline $7000-10000 \mathrm{~m}$ & 5 \\
\hline$>10000 \mathrm{~m}$ & 6 \\
\hline
\end{tabular}

Tabla 6

VALORES UTILIZADOS EN LA ESTANDARIZACIÓN DEL NIVEL FREÁTICO. RANGOS ESTABLECIDOS, EN FUNCIÓN DE LA RENTABILIDAD DE EXTRACCIÓN, TRAS DIVERSAS CONVERSACIONES CON EXPERTOS EN EL TEMA DEL

AYUNTAMIENTO DE ZARAGOZA, GOBIERNO DE ARAGÓN Y CONFEDERACIÓN HIDROGRÁFICA DEL EBRO

\begin{tabular}{|l|c|}
\hline \multicolumn{1}{|c|}{ Valor original } & Valor estandarizado \\
\hline $0-7 \mathrm{~m}$ & 1 \\
\hline $7-10 \mathrm{~m}$ & 4 \\
\hline $10-15 \mathrm{~m}$ & 5 \\
\hline$>15 \mathrm{~m}$ & 6 \\
\hline Zonas fuera del modelo & 6 \\
\hline
\end{tabular}

Tabla 7

VALORES UTILIZADOS EN LA ESTANDARIZACIÓN DE LA LOCALIZACIÓN DEL RECURSO. RANGOS ESTABLECIDOS TRAS DIVERSAS CONVERSACIONES CON EXPERTOS EN EL TEMA DEL AYUNTAMIENTO DE ZARAGOZA, GOBIERNO DE ARAGÓN Y CONFEDERACIÓN HIDROGRÁFICA DEL EBRO. CRITERIO INCREMENTO DE RENTABILIDAD DE EXTRACCIÓN Y REDUCCIÓN DE IMPACTO AMBIENTAL AL INCREMENTAR EL ESPESOR DEL MATERIAL

\begin{tabular}{|l|c|}
\hline \multicolumn{1}{|c|}{ Valor original } & Valor estandarizado \\
\hline $0-5 \mathrm{~m}$ & 1 \\
\hline $5-7 \mathrm{~m}$ & 4 \\
\hline $7-10 \mathrm{~m}$ & 5 \\
\hline$>10 \mathrm{~m}$ & 6 \\
\hline Zonas fuera del modelo & 1 \\
\hline
\end{tabular}

Tabla 8

VALORES UTILIZADOS EN LA ESTANDARIZACIÓN DEL ESPESOR DEL MATERIAL SOBRANTE. RANGOS ESTABLECIDOS, EN FUNCIÓN DE LA RENTABILIDAD ECONÓMICA, TRAS DIVERSAS CONVERSACIONES CON EXPERTOS EN EL TEMA DEL AYUNTAMIENTO DE ZARAGOZA, GOBIERNO DE ARAGÓN Y CONFEDERACIÓN HIDROGRÁFICA DEL EBRO

\begin{tabular}{|l|c|}
\hline \multicolumn{1}{|c|}{ Valor original } & Valor estandarizado \\
\hline $0-3 \mathrm{~m}$ & 6 \\
\hline $3-5 \mathrm{~m}$ & 4 \\
\hline $5-10 \mathrm{~m}$ & 2 \\
\hline$>10 \mathrm{~m}$ & 1 \\
\hline Zonas fuera del modelo & 1 \\
\hline
\end{tabular}




\section{Análisis de alternativa de extracción óptima}

El principal objetivo del análisis de alternativa óptima es la clasificación de las alternativas en función de su preferencia. En este caso las alternativas de localización de futuras zonas de extracción fueron proporcionadas por el Departamento de Medioambiente del Ayuntamiento de Zaragoza, aunque, el reducido número de las mismas, únicamente 3, condicionó la inclusión en el análisis de nuevas zonas ficticias hasta un total de doce alternativas. Desde el punto de vista geométrico estas alternativas están representadas por polígonos.

En esta aproximación no es necesaria la estandarización de los valores asignados a los criterios ya que las alternativas son directamente comparadas por pares en función de sus valores originales para asignar la función de preferencia. Los limitantes y los criterios son los mismos utilizados en el análisis de localización óptima. Sin embargo, existen algunas alternativas que se localizan en zonas que están parcialmente restringidas ya que los polígonos que las delimitan son cruzados por carreteras o vías pecuarias. Este hecho ha implicado la introducción en el análisis de los limitantes como un nuevo criterio, de manera que las zonas con limitación de uso obtienen el valor cero, mientras que las zonas sin restricciones obtienen el valor uno.

Además, es importante definir si el incremento en un criterio en particular implica un incremento o descenso en la idoneidad de la alternativa para el uso. Por ejemplo, en el caso del criterio que representa la cantidad de recurso disponible, un incremento en el criterio implicará una mayor idoneidad de la alternativa. Por el contrario en el caso del nivel freático, un incremento en el mismo implicará una menor idoneidad de la alternativa.

Como se ha mencionado con anterioridad, desde el punto de vista geométrico, cada alternativa es un polígono que contiene diferentes valores para un mismo criterio (pixeles en la capa ráster). Por lo tanto, es necesario seleccionar un único valor para cada alternativa, por lo que se desarrolló una extensión para SIG que permite la selección de diferentes estadísticos del total de la población de datos que están incluidos en cada polígono que define cada alternativa (máximo, mínimo, media, etc.). En nuestro caso, el valor medio fue seleccionado para llevar a cabo el análisis ya que consideramos que este valor representa mejor todos los valores de la alternativa. Por el contrario, los valores mínimos y máximos son normalmente eventos raros con escasa probabilidad de ocurrencia.

La metodología PROMETHEE-2 utiliza la función de preferencia Pj (a,b), la cual es una función de la diferencia entre dos alternativas para un determinado criterio (Brans et al., 1986). Se han propuesto seis tipos de funciones basadas en la noción de criterio. Para un mayor detalle sobre las funciones de preferencia se puede consultar Marinoni (2005), Lamelas (2007) y Lamelas et al. (2010). En este trabajo se ha utilizado la función de preferencia «usual criterion» que se basa en la simple diferencia en los valores asignados a las alternativas ya que esta función ayuda en mayor medida a discriminar entre las alternativas disponibles.

La comparación por pares de alternativas produce una matriz de preferencia para cada criterio (Figura 3). Una vez calculada la matriz de preferencia para cada criterio, se realiza una primera agregación, multiplicando cada valor de preferencia por un factor de peso, w, que expresa el peso o importancia de cada criterio y sumando estos productos (Marinoni, 2005). El resultado es el índice de preferencia, П (ver Figura 3). La metodología AHP ha sido integrada en la herramienta para la asignación de pesos. 


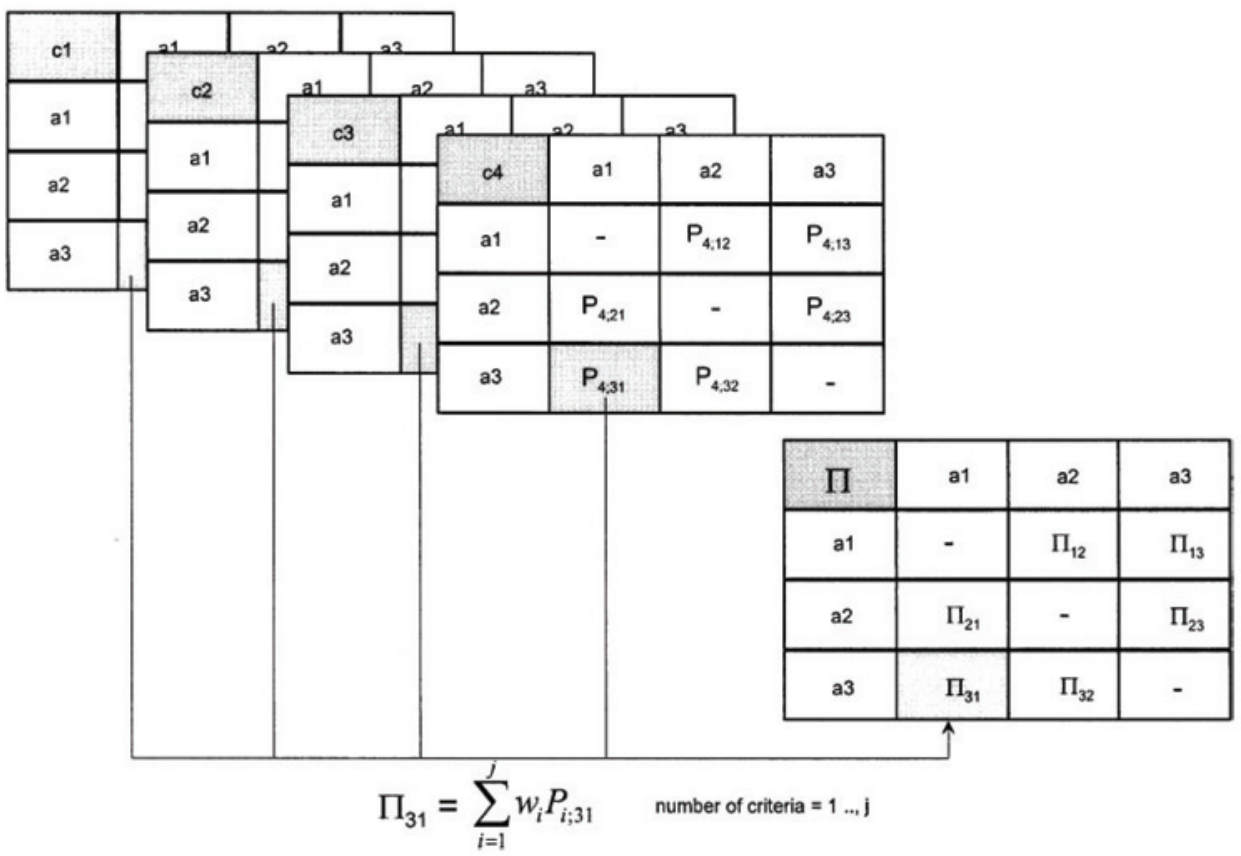

Fuente: Marinoni (2005).

La clasificación final de idoneidad de las alternativas se realiza calculando el flujo neto $\Phi$ (a1) para cada alternativa, a, que se obtiene de la resta del flujo saliente y entrante (leaving flow y entering flow en la literatura inglesa). Cuanto mayor es el flujo neto, mayor es la preferencia de una alternativa sobre las demás (Tabla 9). El flujo saliente $\Phi+$ (a1), representa la media en que una alternativa supera a las demás en idoneidad, mientras que el flujo entrante $\Phi$ - (a1) representa la peor idoneidad de la alternativa con respecto a las demás.

Tabla 9

EJEMPLO HIPOTÉTICO DE ÍNDICE DE PREFERENCIA, FLUJO ENTRANTE $\varnothing+Y$ SALIENTE $\Phi$-, FLUJO NETO $\varnothing$ Y CLASIFICACIÓN FINAL

\begin{tabular}{|c|c|c|c|c|c|c|}
\hline$\Pi$ & $\mathbf{a 1}$ & $\mathbf{a 2}$ & $\mathbf{a 3}$ & $\Phi+(\mathbf{a x})$ & $\Phi(\mathbf{a x})$ & Clasificación \\
\hline $\mathbf{a 1}$ & - & 0.25 & 0.75 & 1.0 & 0 & 2 \\
\hline $\mathbf{a 2}$ & 0.75 & - & 0.75 & 1.5 & 1 & 1 \\
\hline $\mathbf{a 3}$ & 0.25 & 0.25 & - & 0.5 & -1 & 3 \\
\hline$\Phi-(\mathbf{a x})$ & 1 & 0.5 & 1.5 & & & \\
\hline
\end{tabular}

Fuente: Modificado a partir de Marinoni (2005) 


\section{PROMETHEE-2 estocástico}

La aproximación estocástica de PROMETHEE-2 requiere la asignación de distribuciones teóricas a los valores de los criterios de cada una de las alternativas. Los modelos de distribución se infieren de las poblaciones resultantes de los valores de los criterios (valores de los pixeles) de cada una de las alternativas (polígonos). El programa utilizado para ajustar los tipos de distribución, en función del test de ajuste más adecuado, ha sido @ Risk (Palisade Corporation, 2002). Para asignar los tipos de distribución a las alternativas, se han utilizado el test de Anderson-Darling para variables categóricas y el test chi ${ }^{2}$ para las continuas. Además, es importante que las distribuciones se ajusten a las propiedades de los datos, de manera que, si los datos no admiten valores negativos (e.g. altura sobre el nivel del mar) esta propiedad quede reflejada, por ejemplo seleccionando una distribución exponencial. Este es el caso de algunas alternativas en el criterio correspondiente al nivel freático.

Con posterioridad, se aplicó la simulación Monte Carlo (MCS) con un número de iteraciones $n$ de 5.000, produciendo $n$ valores a introducir en la matriz de entrada de datos (Marinoni, 2005).

La Figura 4 muestra el principio de un ciclo de iteración. Siendo $n$ igual a 5.000, el análisis multicriterio se repite 5.000 veces. El resultado se utiliza entonces para conocer las clasificaciones obtenidas por una determinada alternativa o bien analizar las alternativas que han obtenido una determinada clasificación.

Figura 4

DETERMINACIÓN DE VALORES DE ENTRADA EN PROMETHEE PARA CADA CICLO DE ITERACIÓN

\begin{tabular}{|c|c|c|c|c|}
\hline & Criterion 1 & Criterion 2 & $\ldots$ & Criterion $n$ \\
\hline Alternative 1 & 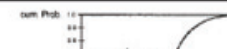 & $\cdot]$ & ... & Distribution $1 n$ \\
\hline Alternative 2 & & & ... & Distribution $2 n$ \\
\hline$\ldots$ & $\ldots$ & $\ldots$ & $\ldots$ & $\ldots$ \\
\hline Alternative $m$ & anso :- & 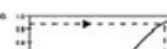 & $\ldots$ & Distribution $\mathrm{mm}$ \\
\hline
\end{tabular}

Fuente: Marinoni (2005).

Sin embargo, la alternativa que obtiene el primer puesto en la clasificación en la mayoría de repeticiones puede no ser necesariamente la mejor. Por esta razón, se sugiere calcular un índice estocástico medio, adimensional MSR para cada una de las alternativas.

$$
\operatorname{MSR} A j, m=\frac{1}{n} \sum_{i=1}^{n}(R i * i) \forall j=1, \ldots, n
$$


donde:

m: número de iteraciones

$A_{j}: j^{\text {th }}$ alternativa

n: número de alternativas disponibles

$\mathrm{R}_{\mathrm{i}}$ : número de clasificaciones $\mathrm{i}^{\text {th }}$ contadas

Para poder comparar el índice estocástico medio de simulaciones con diferente número de iteraciones, se debe estandarizar el valor MSR, dando como resultado el índice de clasificación estocástico SI (Marinoni, 2005):

$$
S I A j, m=\frac{M S R A j, m-M S R \min , m}{M S R \max , m-M S R \min , m}
$$

donde:

m: número de iteraciones

$\mathrm{SI}_{\mathrm{Aj}}$ : índice de clasificación estocástico para la alternativa $\mathrm{j}^{\text {th }}$

$\operatorname{MSR}_{\mathrm{Aj}}$ : MSR para la alternativa jth

$\operatorname{MSR}_{\text {min }}$ : el mínimo valor de MSR

MSR $_{\max }$ : el máximo valor de MSR

Cuanto más próximo a 0 está el valor de SI, mejor es la alternativa.

\section{RESULTADOS Y VALIDACIÓN}

\section{Análisis de localización óptima}

Los valores de preferencia más elevados (y por lo tanto los mayores pesos) han sido asignados a la localización del recurso (valor $0,286=28 \%$ ), protección del acuífero, protección de zonas naturales, así como a zonas con buena capacidad agrícola y mayor profundidad del nivel freático (Tabla 10).

Tabla 10

MATRIZ DE COMPARACIÓN POR PARES Y PESOS DE LOS CRITERIOS PARA EL ANÁLISIS DE LOCALIZACIÓN ÓPTIMA BAJO UN DESARROLLO SOSTENIBLE. A. DISTANCIA EXTRACCIONES, B. PROFUNDIDAD NIVEL FREÁTICO, C. PROTECCIÓN ACUIIFERO, D. DISTANCIA NÚCLEOS, E. DISTANCIA ZONAS PROTEGIDAS, F. DISTANCIA CARRETERAS, G. ESPESOR RECURSO, H. CAPACIDAD AGRÍCOLA, I. ESPESOR MATERIAL SOBRANTE

\begin{tabular}{|c|c|c|c|c|c|c|c|c|c|c|}
\hline $\begin{array}{c}\text { Matriz de } \\
\text { preferencia }\end{array}$ & $\mathbf{A}$ & $\mathbf{B}$ & $\mathbf{C}$ & $\mathbf{D}$ & $\mathbf{E}$ & $\mathbf{F}$ & $\mathbf{G}$ & $\mathbf{H}$ & $\mathbf{I}$ & Pesos \\
\hline $\mathbf{A}$ & 1,00 & 0,20 & 0,14 & 0,33 & 0,14 & 0,50 & 0,12 & 0,20 & 1,00 & 0,022 \\
\hline $\mathbf{B}$ & 5,00 & 1,00 & 0,50 & 4,00 & 0,50 & 4,00 & 0,33 & 1,00 & 5,00 & 0,111 \\
\hline $\mathbf{C}$ & 7,00 & 2,00 & 1,00 & 6,00 & 1,00 & 6,00 & 0,50 & 2,00 & 7,00 & 0,187 \\
\hline $\mathbf{D}$ & 3,00 & 0,25 & 0,16 & 1,00 & 0,16 & 2,00 & 0,14 & 0,25 & 3,00 & 0,043 \\
\hline $\mathbf{E}$ & 7,00 & 2,00 & 1,00 & 6,00 & 1,00 & 6,00 & 0,50 & 2,00 & 7,00 & 0,187 \\
\hline $\mathbf{F}$ & 2,00 & 0,25 & 0,16 & 0,50 & 0,16 & 1,00 & 0,14 & 0,25 & 2,00 & 0,032 \\
\hline $\mathbf{G}$ & 8,00 & 3,00 & 2,00 & 7,00 & 2,00 & 7,00 & 1,00 & 3,00 & 8,00 & 0,280 \\
\hline $\mathbf{H}$ & 5.00 & 1.00 & 0.50 & 4.00 & 0.50 & 4.00 & 0.33 & 1.00 & 5.00 & 0.111 \\
\hline $\mathbf{I}$ & 1.00 & 0.20 & 0.14 & 0.33 & 0.14 & 0.50 & 0.12 & 0.20 & 1.00 & 0.022 \\
\hline
\end{tabular}


En general, la validación de un modelo consiste en comprobar si la estructura del modelo es adecuada para obtener el fin perseguido y con él se consigue un nivel aceptable de precisión en las predicciones. En el caso de modelos explicativos o predictivos, normalmente esta validación se lleva a cabo comprobando el grado de acuerdo entre los datos arrojados por el modelo y los datos del sistema real (Gómez-Delgado, y Barredo, 2005). Para el caso del análisis de capacidad del terreno para acoger actividades extractivas mediante la aplicación de SDSS, el modelo se ha validado mediante la comprobación de que el mismo sigue las preferencias seleccionadas y aplicadas mediante la asignación de los pesos de los criterios.

La Figura 5 muestra el resultado final del análisis de localización óptima de extracciones de gravas y arenas. En la imagen de la izquierda se muestra el resultado de aplicar el análisis teniendo en cuenta principalmente aspectos geocientíficos y buscando un desarrollo sostenible. En blanco se presentan las zonas con limitaciones de uso que, aunque pueden presentar buenos valores de idoneidad, no pueden ser utilizadas. Las zonas más apropiadas para la localización de extracciones (colores grises más oscuros) se localizan en las terrazas altas y en las terrazas cubiertas por glacis, en las que el recurso disponible es elevado. Además, estas zonas se localizan fuera de espacios con valores naturales importantes y en espacios donde la protección del acuífero es mayor y la capacidad agrícola menor. En realidad, estas zonas estas próximas a las que, en la actualidad, se están explotando y cumplen con las preferencias que se han asignado para la elaboración del modelo, dando indicios de la bondad de los resultados. Las zonas menos apropiadas para este uso, se localizan en las terrazas bajas, donde la protección del acuífero es menor y el nivel freático está próximo a la superficie. Este es el caso de La Alfranca (ver localización en Figura 1), donde existe una gran cantidad de recurso, pero existe una baja protección del acuífero y alguna zona de gran valor natural.

La Tabla 11 muestra los valores asignados en la matriz de preferencia y los pesos de cada criterio en el análisis de la sensibilidad del modelo que da mayor importancia al beneficio económico. En este caso, se ha dado más peso al espesor del material sobrante que los aspectos medioambientales. La cartografía de la derecha de la Figura 5 muestra los resultados obtenidos en esta aproximación. Aunque no existen grandes diferencias

Figura 5

a) ANÁLISIS DE LOCALIZACIÓN ÓPTIMA BAJO UN DESARROLLO SOSTENIBLE, b) ANÁLISIS DE LOCALIZACIÓN ÓPTIMA BAJO UN DESARROLLO ÚNICAMENTE ECONÓMICO. EN AMBAS FIGURAS SE MUESTRA LA LOCALIZACIÓN DE ALTERNATIVAS DEL ANÁLISIS DE ALTERNATIVA ÓPTIMA

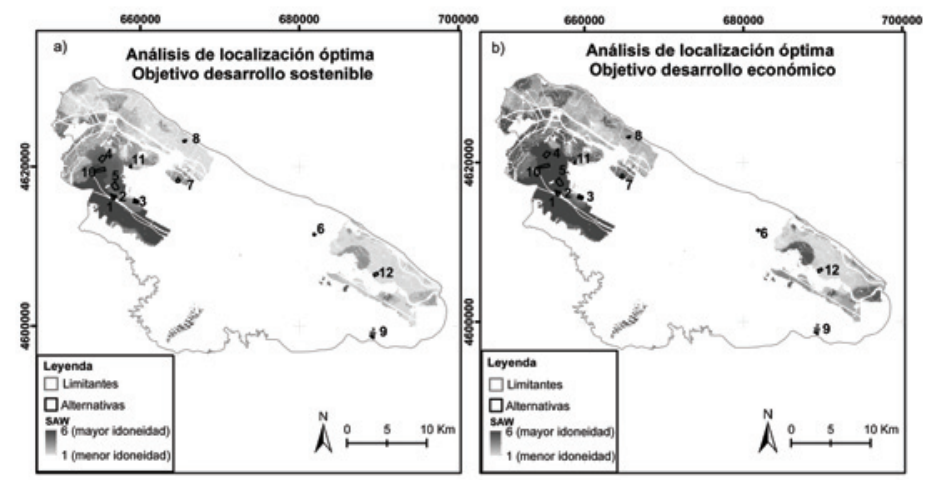


entre los dos modelos, se aprecia como la extensión de zonas apropiadas para la localización de extracciones se ha incrementado, especialmente en las terrazas bajas, ya que la protección del acuífero ha perdido peso en este último análisis. Este es el caso de la Alfranca que presentaba bajos valores de idoneidad debido a la protección del acuífero en el primer análisis.

Tabla 11

MATRIZ DE COMPARACIÓN POR PARES Y PESOS DE LOS CRITERIOS PARA EL ANÁLISIS DE LOCALIZACIÓN ÓPTIMA

PARA UN DESARROLLO ECONÓMICO. A. DISTANCIA EXTRACCIONES, B. PROFUNDIDAD NIVEL FREÁTICO, C. PROTECCIÓN ACUIIFERO, D. DISTANCIA NÚCLEOS, E. DISTANCIA ZONAS PROTEGIDAS, F. DISTANCIA CARRETERAS, G. ESPESOR RECURSO, H. CAPACIDAD AGRÍCOLA SUELOS, I. ESPESOR MATERIAL SOBRANTE

\begin{tabular}{|c|c|c|c|c|c|c|c|c|c|c|}
\hline $\begin{array}{c}\text { Matriz de } \\
\text { preferencia }\end{array}$ & $\mathbf{A}$ & $\mathbf{B}$ & $\mathbf{C}$ & $\mathbf{D}$ & $\mathbf{E}$ & $\mathbf{F}$ & $\mathbf{G}$ & $\mathbf{H}$ & $\mathbf{I}$ & Pesos \\
\hline $\mathbf{A}$ & 1.00 & 0.25 & 3.00 & 1.00 & 3.00 & 1.00 & 0.20 & 2.00 & 0.33 & 0.074 \\
\hline $\mathbf{B}$ & 4.00 & 1.00 & 4.00 & 3.00 & 4.00 & 3.00 & 0.33 & 2.00 & 0.50 & 0.157 \\
\hline $\mathbf{C}$ & 0.33 & 0.25 & 1.00 & 0.33 & 1.00 & 0.33 & 0.17 & 0.50 & 0.25 & 0.033 \\
\hline $\mathbf{D}$ & 1.00 & 0.33 & 3.00 & 1.00 & 3.00 & 1.00 & 0.20 & 2.00 & 0.33 & 0.075 \\
\hline $\mathbf{E}$ & 0.33 & 0.25 & 1.00 & 0.33 & 1.00 & 0.33 & 0.17 & 0.50 & 0.25 & 0.033 \\
\hline $\mathbf{F}$ & 1.00 & 0.33 & 3.00 & 1.00 & 3.00 & 1.00 & 0.20 & 2.00 & 0.33 & 0.075 \\
\hline $\mathbf{G}$ & 5.00 & 3.00 & 6.00 & 5.00 & 6.00 & 5.00 & 1.00 & 4.00 & 2.00 & 0.309 \\
\hline $\mathbf{H}$ & 0.50 & 0.50 & 2.00 & 0.50 & 2.00 & 0.50 & 0.25 & 1.00 & 0.33 & 0.055 \\
\hline $\mathbf{I}$ & 3.00 & 2.00 & 4.00 & 3.00 & 4.00 & 3.00 & 0.50 & 3.00 & 1.00 & 0.187 \\
\hline
\end{tabular}

Para medir el grado de similitud entre los resultados obtenidos en los dos análisis realizados, se ha obtenido el coeficiente de correlación de Pearson entre los valores de las dos imágenes ráster, obteniendo como resultado un valor de 0.713 , con un nivel de significación de 0,01 , que indica un alto grado de ajuste entre los dos resultados.

\section{Análisis de alternativa óptima}

Como se ha comentado en la sección 2.2.2., en el análisis de alternativa óptima se debe incluir un criterio más correspondiente a los limitantes, por lo que los pesos de los criterios asignados en el análisis de localización óptima no pueden ser utilizados, teniendo que ser nuevamente calculados utilizando la metodología AHP. La Tabla 12 muestra la matriz de preferencia y los nuevos pesos asignados a los criterios. Los valores de preferencia de los criterios son similares a los del análisis de localización óptima bajo un concepto de desarrollo sostenible (Tabla 10), pero asignando un mayor peso al factor limitantes o restricciones de uso, para evitar la selección de alternativas incluidas parcialmente en zonas restringidas. 
Tabla 12

MATRIZ DE COMPARACIÓN POR PARES Y PESOS DE LOS CRITERIOS PARA EL ANÁLISIS DE ALTERNATIVA ÓPTIMA.

A. DISTANCIA EXTRACCIONES, B. PROFUNDIDAD NIVEL FREÁTICO, C. PROTECCIÓN ACUÍFERO, D. DISTANCIA NÚCLEOS, E. DISTANCIA ZONAS PROTEGIDAS, F. DISTANCIA CARRETERAS, G. ESPESOR RECURSO, H. CAPACIDAD AGRÍCOLA SUELOS, I. ESPESOR MATERIAL SOBRANTE, J. LIMITANTES

\begin{tabular}{|c|c|c|c|c|c|c|c|c|c|c|c|}
\hline $\begin{array}{c}\text { Matriz de } \\
\text { preferencia }\end{array}$ & $\mathbf{A}$ & $\mathbf{B}$ & $\mathbf{C}$ & $\mathbf{D}$ & $\mathbf{E}$ & $\mathbf{F}$ & $\mathbf{G}$ & $\mathbf{H}$ & $\mathbf{I}$ & $\mathbf{J}$ & Pesos \\
\hline $\mathbf{A}$ & 1.00 & 0.20 & 0.14 & 0.33 & 0.14 & 0.50 & 0.13 & 0.20 & 1.00 & 0.11 & 0.018 \\
\hline $\mathbf{B}$ & 5.00 & 1.00 & 0.50 & 4.00 & 0.50 & 4.00 & 0.33 & 1.00 & 5.00 & 0.20 & 0.079 \\
\hline $\mathbf{C}$ & 7.00 & 2.00 & 1.00 & 6.00 & 1.00 & 6.00 & 0.50 & 2.00 & 7.00 & 0.33 & 0.131 \\
\hline $\mathbf{D}$ & 3.00 & 0.25 & 0.17 & 1.00 & 0.17 & 2.00 & 0.14 & 0.25 & 3.00 & 0.13 & 0.032 \\
\hline $\mathbf{E}$ & 7.00 & 2.00 & 1.00 & 6.00 & 1.00 & 6.00 & 0.50 & 2.00 & 7.00 & 0.33 & 0.131 \\
\hline $\mathbf{F}$ & 2.00 & 0.25 & 0.17 & 0.50 & 0.17 & 1.00 & 0.14 & 0.25 & 2.00 & 0.13 & 0.025 \\
\hline $\mathbf{G}$ & 8.00 & 3.00 & 2.00 & 7.00 & 2.00 & 7.00 & 1.00 & 3.00 & 8.00 & 0.50 & 0.195 \\
\hline $\mathbf{H}$ & 5.00 & 1.00 & 0.50 & 4.00 & 0.50 & 4.00 & 0.33 & 1.00 & 5.00 & 0.20 & 0.079 \\
\hline $\mathbf{I}$ & 1.00 & 0.20 & 0.14 & 0.33 & 0.14 & 0.50 & 0.13 & 0.20 & 1.00 & 0.11 & 0.018 \\
\hline $\mathbf{J}$ & 9.00 & 5.00 & 3.00 & 8.00 & 3.00 & 8.00 & 2.00 & 5.00 & 9.00 & 1.00 & 0.292 \\
\hline
\end{tabular}

En la Tabla 13 se presentan los indices de preferencia y el flujo entrante y saliente generados por la metodología PROMETHEE-2. La Figura 5 muestra la localización de las alternativas incluidas en el análisis. Las alternativas 1, 2 y 3 son las reales, proporcionadas por Ayuntamiento de Zaragoza. Con la excepción de la alternativa 3, las alternativas reales son las que presentan mayor idoneidad. La menor idoneidad de la alternativa 3 puede deberse al menor espesor del recurso y gran espesor del material sobrante existente en la zona donde se localiza. Además, es importante señalar la similitud con los resultados obtenidos en el análisis de localización óptima, ya que las alternativas mejor clasificadas se localizan en las terrazas altas, en algunos casos cubiertas por glacis, que también presentaban altos valores de idoneidad en el análisis de localización óptima, asegurando la robustez de los resultados.

Tabla 13

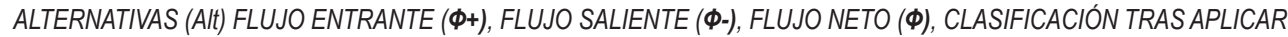
PROMETHEE-2, ÍNDICE DE CLASIFICACIÓN ESTOCÁSTICO (SI), CLASIFICACIÓN TRAS APLICAR PROMETHEE-2 ESTOCÁSTICO Y VALOR OBTENIDO TRAS EL ANÁLISIS DE LOCALIZACIÓN ÓPTIMA (SAW) PARA CADAALTERNATIVA

\begin{tabular}{|c|c|c|c|c|c|c|c|}
\hline Alt & $\mathbf{\Phi +}$ & $\boldsymbol{\Phi}-$ & $\boldsymbol{\Phi}$ & $\begin{array}{c}\text { Clasificación } \\
\text { PROMETHEE }\end{array}$ & $\mathbf{S I}$ & $\begin{array}{c}\text { Clasificación } \\
\text { estocástica }\end{array}$ & $\begin{array}{c}\text { SAW } \\
\text { Valor medio }\end{array}$ \\
\hline $\mathbf{1}$ & 6,07 & 4,51 & 1,56 & 4 & 0,20 & 3 & 4.60 \\
\hline $\mathbf{2}$ & 5,91 & 2,33 & 3,58 & 3 & 0,24 & 4 & 4.58 \\
\hline $\mathbf{3}$ & 4,32 & 3,93 & 0,39 & 8 & 0,62 & 8 & 3.00 \\
\hline $\mathbf{4}$ & 4,50 & 3,85 & 0,64 & 6 & 0,50 & 6 & 3.99 \\
\hline $\mathbf{5}$ & 5,95 & 4,73 & 1,22 & 5 & 0,15 & 2 & 4.27 \\
\hline $\mathbf{6}$ & 1,89 & 8,96 & $-7,07$ & 12 & 1,00 & 12 & 1.57 \\
\hline $\mathbf{7}$ & 4,42 & 3,93 & 0,48 & 7 & 0,54 & 7 & 3.30 \\
\hline $\mathbf{8}$ & 2,07 & 6,43 & $-4,36$ & 11 & 0,89 & 11 & 1.81 \\
\hline $\mathbf{9}$ & 5,99 & 2,36 & 3,64 & 2 & 0,28 & 5 & 4.65 \\
\hline $\mathbf{1 0}$ & 6,49 & 2,17 & 4,31 & 1 & 0,06 & 1 & 4.97 \\
\hline $\mathbf{1 1}$ & 3,72 & 4,62 & $-0,90$ & 9 & 0,69 & 9 & 3.88 \\
\hline $\mathbf{1 2}$ & 2,51 & 6,00 & $-3,49$ & 10 & 0,84 & 10 & 1.71 \\
\hline
\end{tabular}




\section{PROMETHEE-2 estocástico}

En la aproximación estocástica, se debe asignar distribuciones a los criterios de cada alternativa y se lleva a cabo una simulación MCS para aplicar la herramienta de EMC, en este caso PROMETHEE-2, lo que implica la repetición del análisis un número específico de veces (5.000 en nuestro caso). Debe tenerse en cuenta que, debido a la variabilidad local/regional, la distribución de un criterio es muy probable que sea diferente para cada una de las alternativas. De este modo, aunque pueda parecer razonable, en un primer momento, el asignar un tipo de distribución a todas las alternativas de un criterio, si los análisis estadísticos indican que existen diferentes distribuciones para un criterio en las diferentes alternativas, entonces deben asignarse diferentes distribuciones a un mismo criterio en las distintas alternativas.

En la Tabla 14 se presentan los tipos de distribuciones asignadas a cada alternativa y criterio en el análisis estocástico de alternativa óptima.

El tipo de distribución utilizada más habitualmente, en el estudio que nos ocupa, para variables continuas, como la distancia a núcleos, es la distribución normal o log-normal. No obstante, también se han utilizado las distribuciones logística y exponencial, en el caso de variables continuas como la protección del acuífero o el espesor del material sobrante.

En el caso de variables categóricas con dos posibles realidades, se ha utilizado la distribución binomial. Cuando la variable categórica presentaba más de dos clases, la utilización de una distribución categórica podría dar problemas, al incluir en el análisis valores que no están presentes en la realidad. Por ejemplo, si una alternativa presenta valores 1 y 4 en el criterio correspondiente a capacidad agrícola y asignamos una distribución categórica, la simulación habría asignado valores 2 y 3 a la alternativa, aunque estos valores no están presentes en la realidad. Por esta razón, en lugar de asignar una distribución, en estos casos, se ha utilizado el porcentaje de casos (valor p en la Tabla 14) en cada categoría para calcular la probabilidad de ocurrencia de cada categoría. Este fue también el caso de algunas variables continuas que presentaban una baja varianza en los valores que complicaba la selección de un tipo de distribución (e.g. alternativa 10 en protección del acuífero). En este caso, se utilizó en el análisis el porcentaje o probabilidad de ocurrencia de cada valor. Finalmente, algunas alternativas presentan un único valor en todos los pixeles que componen la alternativa (valor único en la Tabla).

En la Table 13, se presentan los resultados del análisis estocástico de alternativa óptima utilizando la metodología PROMETHEE-2 estocástica. En general, existen pocas diferencias entre los resultados de PROMETHEE-2 y su aproximación estocástica. De hecho, en las dos aproximaciones la alternativa 10 es la que presenta la primera clasificación y por lo tanto es la alternativa más idónea. El cambio en la clasificación entre aproximaciones de las alternativas 1 y 2 (tercer y cuarto puesto en las dos clasificaciones) es debido a la localización de una parte de la alternativa 1 en zona con limitación de uso, que hace que, al utilizar el valor medio en la aproximación PROMETHEE-2, el valor incluido en el análisis para este criterio sea mayor que 0 en la alternativa 1 y 0 en la 2 , por lo tanto la alternativa 1 es menos idonea que la 2. Por el contrario, en la aproximación estocástica al utilizar la probabilidad y debido a que el porcentaje de valores 0 (no limitaciones de uso) es muy superior al de valores 1 (existencia de limitaciones), y a que el resto de criterios son mejores en la alternativa 1, ésta es clasificada por delante de la alternativa 2. 
Tabla 14

TIPOS DE DISTRIBUCIÓN ASIGNADAS A LAS ALTERNATIVAS EN CADA CRITERIO EN EL ANÁLISIS ESTOCÁSTICO DE ALTERNATIVA ÓPTIMA. AL. ALTERNATIVA, A. DISTANCIA EXTRACCIONES, B. PROFUNDIDAD NIVEL FREÁTICO, C. PROTECCIÓN ACUÍFERO, D. DISTANCIA NÚCLEOS, E. DISTANCIA ZONAS PROTEGIDAS, F. DISTANCIA CARRETERAS, G. ESPESOR RECURSO, H. CAPACIDAD AGRÍCOLA SUELOS, I. ESPESOR MATERIAL SOBRANTE, J. LIMITANTES, LN= LOG-NORMAL, N=NORMAL, LG= LOGÍSTICA, P=PORCENTAJE, E=EXPONENCIAL, U=VALOR ÚNICO, B=BINOMIAL

\begin{tabular}{|c|c|c|c|c|c|c|c|c|c|c|}
\hline Al. & $\mathbf{A}$ & B & C & D & $\mathbf{E}$ & $\mathbf{F}$ & G & H & I & $\mathbf{J}$ \\
\hline 1 & $\ln$ & $\ln$ & $\mathrm{u}$ & $\mathrm{n}$ & $\mathrm{n}$ & $\ln$ & $\mathrm{n}$ & $\mathrm{u}$ & $\mathrm{e}$ & b \\
\hline 2 & $\ln$ & $\mathrm{n}$ & $\mathrm{u}$ & $\ln$ & $\ln$ & $\mathrm{n}$ & $\lg$ & $\mathrm{u}$ & $\mathrm{e}$ & $\mathrm{u}$ \\
\hline 3 & $\mathrm{n}$ & $\ln$ & $\mathrm{u}$ & $\mathrm{n}$ & $\mathrm{n}$ & $\mathrm{n}$ & $\mathrm{n}$ & $\mathrm{u}$ & $\mathrm{n}$ & $\mathrm{u}$ \\
\hline 4 & $\mathrm{n}$ & $\lg$ & $\lg$ & $\mathrm{n}$ & $\ln$ & $\ln$ & $\mathrm{n}$ & $\mathrm{u}$ & $\mathrm{e}$ & $\mathrm{u}$ \\
\hline 5 & $\mathrm{n}$ & $\mathrm{n}$ & $\mathrm{n}$ & $\mathrm{n}$ & $\ln$ & $\mathrm{n}$ & $\mathrm{n}$ & $\mathrm{u}$ & $\mathrm{e}$ & $\mathrm{b}$ \\
\hline 6 & $\mathrm{n}$ & $\mathrm{p}$ & $\lg$ & $\mathrm{n}$ & $\mathrm{n}$ & $\mathrm{n}$ & $\mathrm{n}$ & $\mathrm{u}$ & $\mathrm{n}$ & $\mathrm{u}$ \\
\hline 7 & $\mathrm{n}$ & $\mathrm{n}$ & $\lg$ & $\mathrm{n}$ & $\mathrm{n}$ & $\mathrm{n}$ & $\mathrm{n}$ & $\mathrm{u}$ & $\mathrm{u}$ & $\mathrm{u}$ \\
\hline 8 & $\mathrm{n}$ & $\mathrm{n}$ & $\lg$ & $\ln$ & $\ln$ & $\ln$ & $\lg$ & $\mathrm{u}$ & $\mathrm{n}$ & $\mathrm{u}$ \\
\hline 9 & $\ln$ & $\mathrm{u}$ & $\mathrm{u}$ & $\ln$ & $\mathrm{n}$ & $\mathrm{n}$ & $\mathrm{u}$ & $\mathrm{u}$ & $\mathrm{u}$ & $\mathrm{u}$ \\
\hline 10 & $\mathrm{n}$ & $\lg$ & $\mathrm{p}$ & $\ln$ & $\mathrm{n}$ & $\mathrm{n}$ & $\ln$ & $\mathrm{p}$ & $\mathrm{e}$ & $\mathrm{u}$ \\
\hline 11 & $\ln$ & e & $\lg$ & $\mathrm{n}$ & $\mathrm{n}$ & $\mathrm{n}$ & $\lg$ & $\mathrm{u}$ & $\mathrm{n}$ & $\mathrm{u}$ \\
\hline 12 & $\mathrm{n}$ & $\mathrm{p}$ & $\lg$ & $\mathrm{n}$ & $\mathrm{e}$ & $\mathrm{n}$ & $\lg$ & $\mathrm{u}$ & $\mathrm{n}$ & $\mathrm{u}$ \\
\hline
\end{tabular}

\section{DISCUSIÓN Y CONCLUSIONES}

La cartografía de localización óptima de nuevas zonas de extracción de arenas y gravas desarrollada mediante la integración de las metodologías SAW y AHP en SIG es de gran utilidad para la ordenación de los usos del suelo en los alrededores de Zaragoza. Existe un beneficio adicional alcanzado mediante la integración de aspectos geocientíficos en el proceso de toma de decisiones sobre los usos del suelo de esta ciudad, en busca de un desarrollo más sostenible, tal y como es demandado por la Agenda 21.

Un problema fundamental en la teoría de la decisión es como asignar pesos a un conjunto de criterios de acuerdo con su importancia. La metodología AHP es un excelente y ampliamente conocido método para la evaluación de tierras. Sin embargo, una desventaja del mismo es la inherente subjetividad de la asignación de valores de preferencia entre criterios. Además, en general los pesos derivados de estos valores de preferencia tienen un gran efecto en los resultados obtenidos en un análisis de idoneidad del terreno para un determinado uso del suelo. Sin embargo, en el caso particular de la localización de nuevas zonas de extracción, los resultados no han variado mucho al aplicar el análisis de idoneidad bajo un concepto de desarrollo sostenible y de desarrollo más económico, aunque los pesos asignados a los criterios se hayan modificado de una aproximación a otra. No obstante, este no es el caso más habitual y, en muchas ocasiones, los resultados varían más al modificar los pesos de los criterios, por lo que, una posible solución al problema de asignación de pesos, podría 
venir por el establecimiento de valores de preferencia entre criterios por diferentes agentes de la decisión, de manera que se creasen diferentes cartografías de localización óptima de un determinado uso del suelo, para, finalmente, combinar todas y seleccionar la mejor localización desde todos los puntos de vista.

Tras entablar varias conversaciones con expertos en el tema del Ayuntamiento de Zaragoza, el Gobierno de Aragón y la Confederación Hidrográfica del Ebro y buscando un desarrollo sostenible, los resultados del análisis de localización óptima nos indican que las zonas más apropiadas para la localización de extracciones son las terrazas altas y las terrazas cubiertas por glacis, en las que el recurso disponible es elevado. Además, estas zonas se localizan fuera de espacios con valores naturales importantes y en espacios donde la protección del acuífero es mayor y la capacidad agrícola menor. Las zonas menos apropiadas para este uso, se localizan en las terrazas bajas donde la protección del acuífero es menor y el nivel freático está próximo a la superficie.

Una de las ventajas de las técnicas de concordancia como PROMETHEE-2 es la ausencia de necesidad de estandarizar las variables, lo que en definitiva reduce la subjetividad. Sin embargo, en un análisis espacial de EMC existen varias decisiones que deben ser tomadas, como, por ejemplo, que valor de los presentes en la alternativa escoger, de entre la población de pixeles que la conforman, para llevar a cabo el análisis posterior (e.g. máximo, mínimo, media, etc.). Además, en el caso de aplicar la metodología PROMETHEE-2, otra decisión que se debe tomar es el tipo de función de preferencia a aplicar, así como los pesos a asignar a los criterios.

Es de resaltar la similitud entre resultados obtenidos en el análisis de localización óptima y los resultados del análisis de alternativa óptima. En general, las alternativas con mejores clasificaciones en el análisis de alternativas óptimas se localizan en las zonas con valores de mayor idoneidad en el análisis de localización óptima. Se observan algunas diferencias como es el caso de las alternativas 1 y 2, que varían su clasificación debido a que en el análisis de alternativa óptima los limitantes se han introducido como un criterio más.

En el análisis de alternativa óptima, si al aplicar PROMETHEE-2 se utiliza el valor medio de la población de pixeles que conforman la alternativa, se obtiene un resultado medio en el que no se puede evaluar la incertidumbre de los valores de entrada ni de los resultados. La aproximación estocástica utilizada en este trabajo, ayuda a resolver este problema mediante la asignación de distribuciones de probabilidad a los valores de entrada, en lugar de un único valor. Por este motivo, es nuestra recomendación el utilizar aproximaciones estocásticas cuando se trabaja en un entorno de análisis multicriterio de datos y variables espaciales, aunque, en este caso, el proceso no esté absolutamente integrado en el SIG, y por lo tanto sea más tedioso.

\section{AGRADECIMIENTOS}

Esta investigación ha sido posible gracias a la subvención del Servicio de Investigación Alemán (DFG, Ho 804/7-1+2) y a la colaboración del Departamento de Geografía y Ordenación del Territorio de la Universidad de Zaragoza. Es necesario dar las gracias a los directores y principal colaborador de este trabajo, Prof. Dr. Andreas Hoppe, Dr. Juan de la Riva y Oswald Marinoni por sus aportaciones en la investigación. Nos gustaría dar las gracias a la 
Confederación Hidrográfica del Ebro, el Gobierno de Aragón, el Ayuntamiento de Zaragoza, por la información que nos proporcionaron y en especial a los técnicos de dichas Instituciones que colaboraron en la selección de criterios y asignación de pesos de los mismos.

\section{REFERENCIAS}

AMADIO, V., AMADEI, M., BAGNAIA, R., DI BUCCI, D., LAURETI, L., LISI, A., LUGERI, F.R., LUGERI, N. (2002): «The role of geomorphology in Landscape Ecology: the Landscape Unit Map of Italy, Scale 1:250,000 (Carta Della Natura Project)» en Applied Geomorphology: Theory and Practice (Allison, R.J. ed). Chichester, John Wiley and Sons Ltd.

BANAI, R. (1993): «Fuzziness in geographical information systems: contributions from the analytic hierarchy process». International Journal Geographical Information Systems, $\mathrm{n}^{\circ}$ 7(4), 315-329.

BANAI-KASHANI, R. (1989): «A new method for site suitability analysis: the analytical hierarchy process». Environmental management, nº 13, 685-693.

BARREDO, J. y BOSQUE, J. (1995): «Integración de evaluación multicriterio y sistemas de información geográfica para la evaluación de la capacidad de acogida del territorio y la asignación de usos del suelo» en Actas del IV Congreso Español de Sistemas de información Geográfica (AESIG ed.). Barcelona, AESIG, 191-200.

BENITO, G., PÉREZ-GONZALEZ, A., GUTIÉRREZ-SANTOLALLA, F. y MACHADO, M.J. (1998): «River response to Quaternary subsidence due to evaporite solution (Gállego River, Ebro Basin, Spain) ». Geomophology, n 22, 243-263.

BOOTY, W.G., LAM, D.C., WONG, I.W. y SICONOLFI, P. (2001): «Design and implementation of an environmental decision support system». Environmental Modelling and Software, $\mathrm{n}^{\circ} 16,453-458$.

BOSQUE, J. y FRANCO, S. (1995): «Modelos de localización-asignación y evaluación multicriterio para la localización de instalaciones no deseables». Serie Geográfica, n5, 97-112.

BOSQUE, J. y GARCÍA, R.C. (2000): «El uso de los sistemas de información geográfica en la planificación territorial». Anales de Geografía de la Universidad Complutense, $\mathrm{n}^{\mathrm{o}} 20$, 49-67.

BOSQUE, J. y MORENO, A. (2004.): Sistemas de información geográfica y localización óptima de instalaciones y equipamientos. Madrid, RA-MA.

BOSQUE, J., DIAZ, M.A., GÓMEZ, M., RODRÍGUEZ, V.M., RODRÍGUEZ, A.E. y VELA, A. (1999): «Un procedimiento basado en un SIG, para localizar centros de tratamiento de residuos». Anales de Geografía de la Universidad Complutense, n 9, 295-323.

BRANS, J.P., VINCKE, P. y MARESCHAL, B. (1986): «How to select and how to rank projects: The PROMETHEE method». European Journal of Operational Research, $\mathrm{n}^{\circ} 4$, 228-238.

CAN, A. (1993): «Residential quality assessment, Alternative approaches using GIS» en Geographic information systems, spatial modelling and policy evaluation (Fischer M. y Nijkamp P. eds.). Berlín, Springer-Verlang.

CARVER, S.J. (1991): «Integrating multicriteria evaluation with geographical information systems». International Journal of Geographical Information Systems, n 5, 321-339. 
CARVER, S.J. (1999): «Developing Web-based GIS/MCE: Improving access to data and spatial decision support tools» en Multicriteria decision-making and analysis: A geographic information science approach (Thill J.C. eds). New York, Ashgate, 49-76.

DE LA ROSA, D. y MAGALDI, D. (1982): Rasgos metodológicos de un sistema de evaluación de tierras para regiones mediterráneas. Madrid. Sociedad Española de la Ciencia del Suelo.

EASTMAN, J.R. (1997): IDRISI for Windows, Version 2.0: Tutorial exercises. Graduated School of Geography. Worcester. Clark Unversity.

EASTMAN, J.R., KYEM, P.A., TOLEDANO, J. y JIN W. (1993): Gis and Decision Making. Ginebra. United Nations institute for Training and Research.

GÓMEZ-DELGADO, M. y BARREDO, J.I. (2005): Sistemas de Información Geográfica y evaluación multicriterio en la ordenación del territorio. Paracuellos de Jiloca. RA-MA Editorial.

HEVELINK, G.B.M., BURROUGH, P.A. y STEIN, A. (1989): «Propagation of errors in spatial modelling with GIS». International Journal of Geographical Information Systems, $n^{\circ} 3(4), 303-322$.

HEYWOOD, I., CORNELIUS, S. y CARVER, S. (2002): An introduction to geographical information Systems. Harlow. Prentice Hall.

HÖLTING, B., HAERTLÉ, T., HOHBERGER, K.-H., NACHTIGALL, K.H., VILLINGER, E., WEINZIERL, W. y WROBEL, J.-P. (1995): «Konzept zur Ermittlung der Schutzfunktion der Grundwasserüberdeckung». Geologisches Jahrbuch C; n 63, 5-24.

JANKOWSKI, P. (1995): «Integrating geographical information systems and multiple criteria decision-making». International Journal of Geographical Information Systems, $\mathrm{n}^{\circ}$ 9, 251-273.

JANSSEN, R. y RIETVELD, P. (1990): «Multicriteria analysis and GIS: An Application to Agricultural Land-use in the Netherlands» en Geographical Information Systems for Urban and Regional Planning (Scholten H. y Stillwell J. eds.). Dordrecht. Kluwer.

LAMELAS, M.T. (2007): Geo-resources and geo-hazards in the context of a sustainable development in the periphery of urban areas, exemplary of a part of the Ebro Basin in the surroundings of Zaragoza (Spain). Tesis doctoral. Technische Universität Darmstadt. Disponible en http://elib.tu-darmstadt.de/diss/000794 (último acceso Agosto de 2012).

LAMELAS, M.T., MARINONI, O., DE LA RIVA, J. y HOPPE, A. (2010): «Sustainable spatial decision making in a complex $3 \mathrm{~d}$ environment: an auditable decision support workflow based on multicriteria analysis» en Geomorphology: Processes, Taxonomy and Applications (Series: Earth Sciences in the 21st Century (Sanders M.H. and Clark Ph.D. eds.). New York. Nova Science Publishers, 1-44.

LODWICK, W.A., MONSON, W. y SVOBODA, L. (1990): «Attribute error and sensitivity analysis of map operations in geographical information systems: suitability analysis». International Journal of Geographical Information Systems, $\mathrm{n}^{\circ}$ 4(4), 413-428.

MALCZEWSKI, J. (1996): «A GIS-based approach to multiple criteria group decisionmaking». International Journal of Geographical Information Systems, ${ }^{\circ} 10(8), 955-971$. MALCZEWSKI, J. (1999): GIS and Multicritera decision analysis. New York. Wiley;.

MALCZEWSKI, J. (2004): «GIS-based land-use suitability analysis: a critical overview». Progress in Planning, $\mathrm{n}^{\circ}$ 62, 3-65. 
MALCZEWSKI, J. y RINNER, C. (2005): «Exploring multicriteria decision strategies in GIS with linguistic quantifiers: A case study of residential quality evaluation». Journal of Geographical Systems, n' 7, 249-268.

MALCZEWSKI, J., MORENO-SÁNCHEZ, R., BOJORQUEZ-TAPIA, L.A. y ONGAYDELHUMEAU, E. (1997): «Environmental conflict analysis in the Cape Region, Mexico». Journal of Environmental Planning and Management, $\mathrm{n}^{\circ}$ 40(3), 349-374.

MARINONI, O. (2004): «Implementation of the analytical hierarchy process with VBA in ArcGIS». Computers and Geosciences, $\mathrm{n}^{\circ}$ 30, 637-646.

MARINONI, O. (2005): «A stochastic spatial decision support system based on PROMETHEE». International Journal of Geographical Information Science, $\mathrm{n}^{\circ}$ 19(1), 51-68.

MILLER, L., COLLINS, M.G., STEINER, F.R. y COOK, E. (1998): «An approach for greenway suitability analysis». Landscape and Urban Planning, n 42, 91-105.

PALISADE CORPORATION. (2002): @ Risk 4.5.2. Advanced risk analysis for spreadsheets. New York: Newfield.

PEREIRA, J.M.C. y DUCKSTEIN, L. (1993): «A multiple criteria decision-making approach to GIS-based land suitability evaluation». International Journal of Geographical Information Systems, $\mathrm{n}^{\mathrm{o}}$ 7, 407-424.

RINNER, C. y MALCZEWSKI, J. (2002): «Web-enabled spatial decision analysis using ordered Weighted averaging (OWA)». Journal of Geographical Systems, nº 4 (4), 385403.

RINNER C. (2003): «Web-based spatial decision support: status and research directions». Journal of Geographic Information and Decision Analysis, ${ }^{\circ}$ 7(1), 14-31.

SAATY, T.L. (1977): «A scaling method for priorities in hierarchical structures». Journal of Mathematical Psychology, $\mathrm{n}^{\mathrm{o}}$ 15, 231-281.

SHIM, J.P., WARKENTIN, M., COURTNEY, J.F., POWER, D.J., SHARDA, R. y CARLSSON, C. (2002): «Past, present and future of decision support technology». Decision Support Systems, n 33, 111-126.

SORIANO M.A. y SIMÓN J.L. (1995): «Alluvial dolines in the central Ebro basin, Spain: a spatial and developmental hazard analysis». Geomorphology, n 11, 295-309.

THILL, J.C. (1999): Spatial Multicriteria Decision Making and Analysis. A geographic information science approach. Aldershot. Ashgate.

VINCKE, P. (1986): «Analysis of multicriteria decision aid in Europe», European Journal of Operational Research, $\mathrm{n}^{\circ} 25,160-168$.

VOOGD, H. (1983): Multicriteria evaluation for urban and regional planning. London: Pion Limited.

WALLENIUS, J., DYER, J.S., FISHBURN, P.C., STEUER, R.E., ZIONTS, S. y DEB, K. (2008): «Multiple Criteria Decision Making, Multiattribute Utility Theory: Recent accomplishments and what lies ahead». Management science, $\mathrm{n}^{\circ} 54$ (7), 1336-1349.

ZHU, X y DALE, A.P. (2001): «A Web-based decision analysis tool for natural resource and environmental management». Environmental Modelling \& Software, n 16, 251-262. 